\title{
The Synthesis of Complex Arithmetic Computation on Stochastic Bit Streams Using Sequential Logic
}

\author{
Peng Li ${ }^{\dagger}$, David J. Lilja ${ }^{\dagger}$, Weikang Qian ${ }^{\ddagger}$, Kia Bazargan ${ }^{\dagger}$, and Marc Riedel ${ }^{\dagger}$ \\ $\dagger$ Department of Electrical and Computer Engineering, University of Minnesota, Twin Cities, USA \\ $\ddagger$ Electrical and Computer Engineering Devision, University of Michigan-Shanghai Jiao Tong University Joint Institute, China \\ \{lipeng, lilja,kia, mriedel\}@umn.edu, qianwk@sjtu.edu.cn
}

\begin{abstract}
The paradigm of logical computation on stochastic bit streams has several key advantages compared to deterministic computation based on binary radix, including error-tolerance and low hardware area cost. Prior research has shown that sequential logic operating on stochastic bit streams can compute non-polynomial functions, such as the tanh function, with less energy than conventional implementations. However, the functions that can be computed in this way are quite limited. For example, high order polynomials and non-polynomial functions cannot be computed using prior approaches. This paper proposes a new finite-state machine (FSM) topology for complex arithmetic computation on stochastic bit streams. It describes a general methodology for synthesizing such FSMs. Experimental results show that these FSM-based implementations are more tolerant of soft errors and less costly in terms of the area-time product that conventional implementations.
\end{abstract}

\section{INTRODUCTION}

Circuit reliability has become increasingly important in recent years [1], [2], [3]. The paradigm of logical computation on stochastic bit stream has become an attractive solution for many applications. It uses conventional digital logic to perform computation on random bit streams, where the signal value is encoded as the probability of a one versus a zero in the stream. This approach can gracefully tolerate very large errors at lower cost than conventional techniques, such as over-design, while maintaining equivalent performance [4]. The images in Fig. 1 for example, illustrated that the fault tolerance capability of stochastic computing for image segmentation that is based on the frame difference algorithm as opposed to a conventional implementation. As the soft error injection rate increases, a conventional implementation of the image segmentation algorithm rapidly degrades until the output image is no longer recognizable. However, the second row shows that an implementation using stochastic bit streams to encode the data is able to produce the correct output at even very high error rates.

In addition, computations on stochastic bit streams can be performed with very simple logic. For example, multiplication can be implemented with an AND gate [6], [7]. Assuming that the two input stochastic bit streams $A$ and $B$ are independent, the number represented by the output stochastic bit stream $C$ is $c=P(C=$ $1)=P(A=1$ and $B=1)=a \cdot b$. So the AND gate multiplies the two values represented by the stochastic bit streams. Scaled addition can be implemented with a multiplexer (MUX) [6], [7], [8]. With the assumption that the three input stochastic bit streams $A, B$, and $S$ are independent, the number represented by the output stochastic

Permission to make digital or hard copies of all or part of this work for personal or classroom use is granted without fee provided that copies are not made or distributed for profit or commercial advantage and that copies bear this notice and the full citation on the first page. To copy otherwise, to republish, to post on servers or to redistribute to lists, requires prior specific permission and/or a fee.

IEEE/ACM International Conference on Computer-Aided Design (ICCAD) 2012, November 5-8, 2012, San Jose, California, USA

Copyright(c)2012 ACM 978-1-4503-1573-9/12/11.. \$15.00 bit stream $C$ is $c=P(C=1)=P(S=1$ and $A=1)+P(S=$ 0 and $B=1)=s \cdot a+(1-s) \cdot b$. Thus the computation performed by a MUX is the scaled addition of the two input values $a$ and $b$, with a scaling factor of $s$ for $a$ and $(1-s)$ for $b$.

Despite its great potential in terms of high fault-tolerance and low hardware cost, stochastic computing suffers from encoding inefficiency. Assume that a numerical value is represented by $M$ bits using binary radix, we need $2^{M}$ bits to represent the same value stochastically. For small values of $M$ such as $M=8$ (e.g., used in most image processing algorithms and artificial neural networks), the benefits of stochastic computing overwhelm its high encoding overhead. In terms of performance, although computation on bit streams needs more clock cycles to finish, the circuit can be operated under a much faster clock frequency. This is because the circuit is extremely simple, and has a much shorter critical path. In addition, computation on bit streams can be implemented in parallel by trading off silicon area with the number of clock cycles [8].

In terms of energy consumption, complex computations on stochastic bit streams can be performed using quite simple sequential logic. Brown and Card [7] showed that, if a single input linear finitestate machine (FSM) is used to perform the exponentiation and tanh functions on stochastic bit streams, when $M \leq 10$ it will consume less energy than deterministic implementations using adders and multipliers based on binary radix.

However, one shortcoming of the work in [7] is that the functions that can be implemented using the proposed FSM are very limited. For example, high order polynomials and non-polynomials such as functions used in low-density parity-check coding cannot be implemented using the FSM introduced in the previous work [7]. As a result, most applications in artificial neural networks (ANNs), communication systems, and digital signal processing, cannot benefit from this technique.

To solve this problem, we find that the FSM used in stochastic computing can be analyzed using Markov chains [7], [9]. By redesigning the topology of the FSM, we increase the degree of design freedom, so that more sophisticated functions can be synthesized stochastically. The remainder of this paper is organized as follows. In Section $\Pi$, we briefly review the previous work in this area. In Section III, we demonstrate the new FSM topology. In Section IV we introduce the synthesis approach. In Section $\mathrm{V}$, we present the results of synthesis trials, comparing cost, fault-tolerance, and energy consumption of the proposed designs with the previous ones. Section VI concludes and discusses future directions.

\section{RELATED WORK}

Logical computation on stochastic bit streams was first introduced by Gaines [6]. He proposed two coding formats: a unipolar format and a bipolar format. Both formats can coexist in a single system. In the unipolar coding format, a real number $x$ in the unit interval (i.e., 


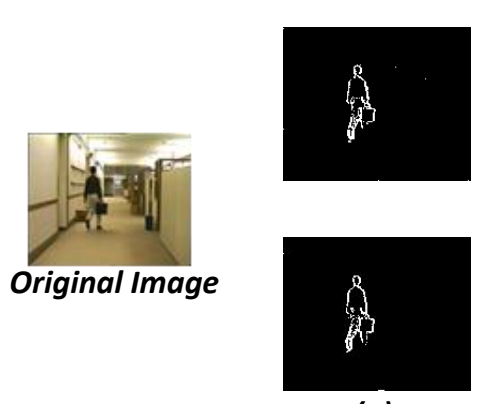

(a)
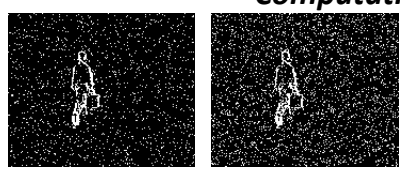

Computation

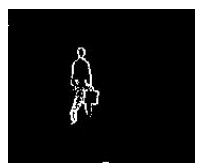

(b)

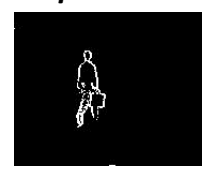

(c)
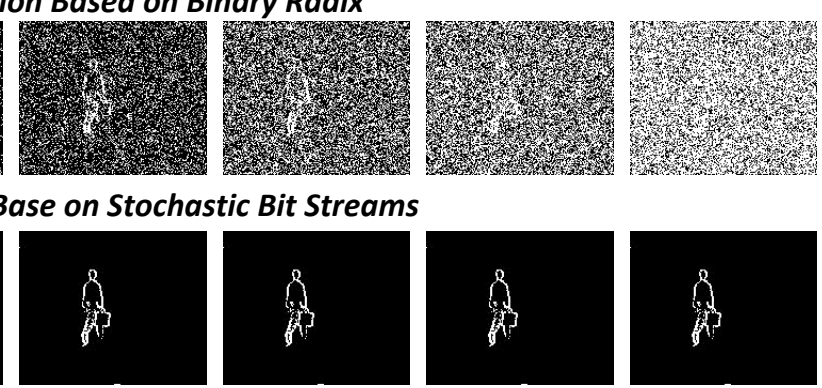

(d)

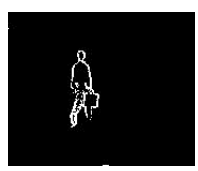

(e)

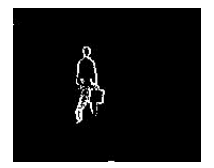

(f)

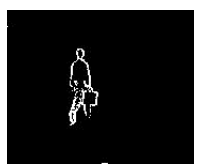

(g)

Fig. 1. A comparison of the fault tolerance capabilities of different hardware implementations for the frame difference based image segmentation algorithm. The images in the first row are generated by a conventional implementation. The images in the second row are generated using a stochastic implementation. Soft errors are injected at a rate of (a) $0 \%$; (b) $1 \%$; (c) $2 \%$; (d) $5 \%$; (e) $10 \%$; (f) $15 \%$; (g) $30 \%$ [5].

$0 \leq x \leq 1)$ corresponds to a sequence of random bits, each of which has probability $x$ of being one and probability $1-x$ of being zero. If a stochastic bit stream of length $N$ has $k$ ones, then the real value represented by the bit stream is $\frac{k}{N}$. In the bipolar coding format, the range of a real number $x$ is extended to $-1 \leq x \leq 1$. The probability that each bit in the stream is one is $P(X=1)=\frac{x+1}{2}$. Thus, a real number $x=-1$ is represented by a stream of all zeros and a real number $x=0$ is represented by a stream of bits that have probability 0.5 of being one. If a stochastic bit stream of length $N$ has $k$ ones, then the real value represented by the bit stream is $2 \frac{k}{N}-1$.

Beginning with the work by Gaines, prior research has described the implementations of specific arithmetic functions based on the stochastic representation [6]. These include constructs for multiplication and addition, discussed in the previous section, as well as constructs for the tanh and the exponentiation functions proposed by Brown and Card [7].

The tanh and the exponentiation functions are implemented with sequential logic, in the form of a single input linear finite state machine [7]. Its state transition diagram is shown in Fig. 2 The machine has a single input $X$ and a set of states $S_{0}, S_{1}, \ldots, S_{N-1}$ arranged as a linear sequence. Given the current state $S_{t}(0<t<N-1)$, the next state will be $S_{t-1}$ if $X=0$ and will be $S_{t+1}$ if $X=1$.

With a stochastic encoding, the input $X$ takes the form of a stochastic bit stream. As a result the state transition process is a special type of a Markov chain [7], [9]. The output $Y$ of this state machine, not shown in Fig. 2, is only determined by the current state: for some states the output $Y$ is one and for the others it is zero. Thus, the output $Y$ is also a stochastic bit stream. Based on different choices of the set of states that let the output $Y$ be one, this linear FSM can be used to implement different functions.

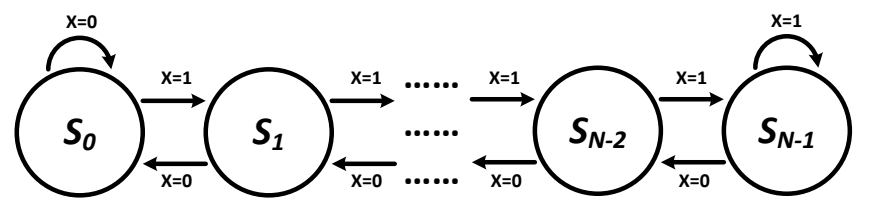

Fig. 2. A generic linear state transition diagram.

For example, consider an FSM whose output $Y$ is one when the current state is in the set $\left\{S_{N / 2}, \ldots, S_{N-1}\right\}$ and is zero when the current state is in the set $\left\{S_{0}, \ldots, S_{N / 2-1}\right\}$, as shown in the state transition diagram in Fig. 3 . If we let $x$ be the bipolar coding of the input bit stream $X$ and $y$ be the bipolar coding of the output bit stream $Y$, then the functional relation between $x$ and $y$ is approximately a tanh function [7],

$$
y \approx \frac{e^{\frac{N}{2} x}-e^{-\frac{N}{2} x}}{e^{\frac{N}{2} x}+e^{-\frac{N}{2} x}} .
$$

Now consider another FSM whose output $Y$ is one when the current state is in the set $\left\{S_{0}, \ldots, S_{N-G-1}\right\}$ and is zero when the current state is in the set $\left\{S_{N-G}, \ldots, S_{N-1}\right\}$ (where $G \ll N$ ), as shown in the state transition diagram in Fig. 4 If we let $x$ be the bipolar coding of the input bit stream $X$ and $y$ be the unipolar coding of the output bit stream $Y$, then the functional relation between $x$ and $y$ is approximately an exponentiation function [7],

$$
y \approx \begin{cases}e^{-2 G x}, & 0 \leq x \leq 1, \\ 1, & -1 \leq x<0 .\end{cases}
$$

However, besides these two functions, how to configure other functions based on the linear FSM has not been studied. In 2008, Qian et al. proposed a general approach for synthesizing combinational logic to implement polynomials on stochastic bit streams [8]. The procedure begins by transforming a power-form polynomial into a Bernstein polynomial [10]. For example, The polynomial

$$
f(x)=\frac{1}{4}+\frac{9}{8} x-\frac{15}{8} x^{2}+\frac{5}{4} x^{3},
$$

can be converted into a Bernstein polynomial of degree 3 :

$$
f(x)=\frac{2}{8} B_{0,3}(x)+\frac{5}{8} B_{1,3}(x)+\frac{3}{8} B_{2,3}(x)+\frac{6}{8} B_{3,3}(x),
$$

where each $B_{i, 3}(x)(0 \leq i \leq 3)$ is a Bernstein basis polynomial of the form

$$
B_{i, 3}(x)=\left(\begin{array}{l}
3 \\
i
\end{array}\right) x^{i}(1-x)^{3-i} .
$$

A Bernstein polynomial,

$$
y=B(x)=\sum_{i=0}^{n} b_{i} B_{i, n}(x),
$$

with all coefficients $b_{i}$ in the unit interval can be implemented stochastically by a generalized multiplexing circuit, shown in Fig. 5 The circuit consists of an adder block and a multiplexer block. The inputs to the adder are $x_{1}, \ldots, x_{n}$. The data inputs to the multiplexer 


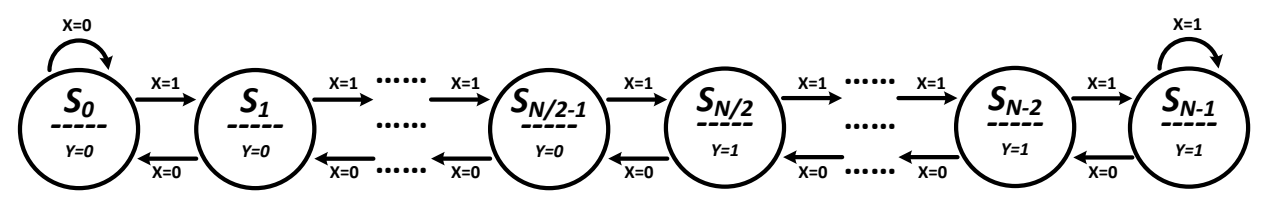

Fig. 3. The state transition diagram of an FSM implementing a tanh function stochastically. In the figure, the number below each state $S_{i}$ represents the output $Y$ of the FSM when the current state is $S_{i}(0 \leq i \leq N-1)$ [7].

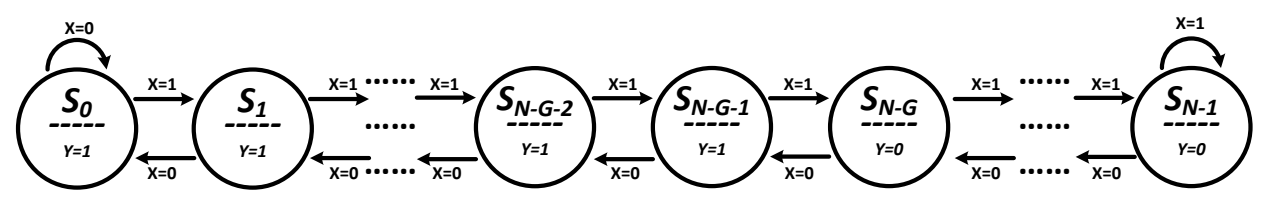

Fig. 4. The state transition diagram of an FSM implementing an exponentiation function stochastically. In the figure, the number below each state $S_{i}$ represents the output $Y$ of the FSM when the current state is $S_{i}(0 \leq i \leq N-1)$. Note that the number $G \ll N[7]$.

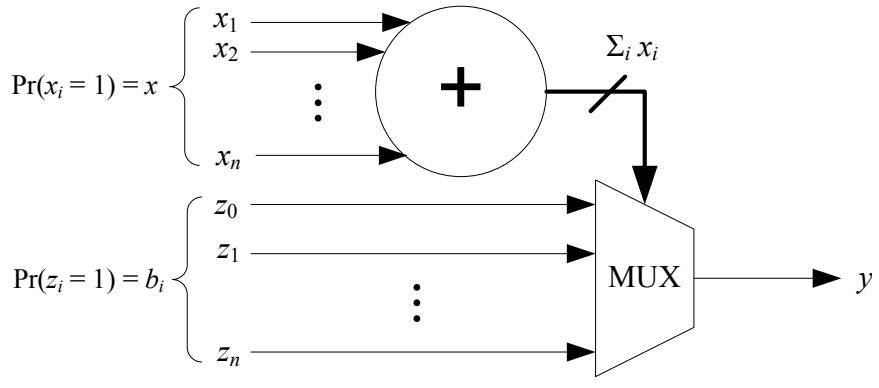

Fig. 5. A generalized multiplexing circuit implementing the Bernstein polynomial $y=B(x)=\sum_{i=0}^{n} b_{i} B_{i, n}(x)$ with $0 \leq b_{i} \leq 1$, for $i=0,1, \ldots, n[8]$

are $z_{0}, \ldots, z_{n}$. The outputs of the adder are the selecting inputs to the multiplexer block.

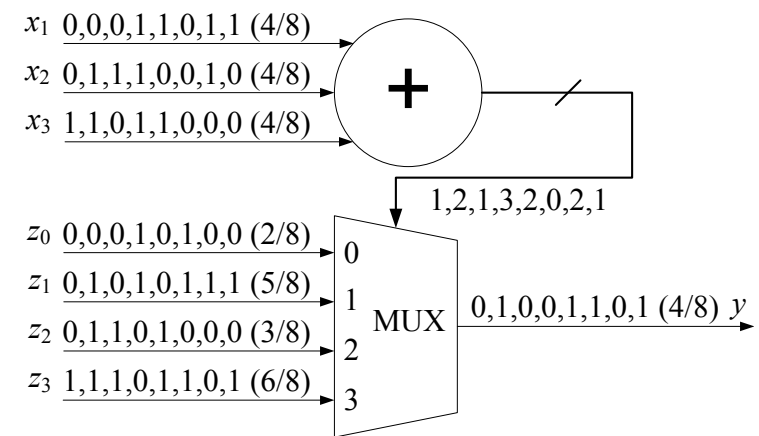

Fig. 6. Logical computation on stochastic bit streams implementing the Bernstein polynomial $f(x)=\frac{2}{8} B_{0,3}(x)+\frac{5}{8} B_{1,3}(x)+\frac{3}{8} B_{2,3}(x)+\frac{6}{8} B_{3,3}(x)$ at $x=0.5$. Stochastic bit streams $x_{1}, x_{2}$ and $x_{3}$ encode the value $x=0.5$. Stochastic bit streams $z_{0}, z_{1}, z_{2}$ and $z_{3}$ encode the corresponding Bernstein coefficients [8].

When the number of ones in the input set $\left\{x_{1}, \ldots, x_{n}\right\}$ is $i$, then the adder will output a binary number equal to $i$ and the output $y$ of the multiplexer will be set to $z_{i}$. The inputs $x_{1}, \ldots, x_{n}$ are independent stochastic bit streams $X_{1}, \ldots, X_{n}$ representing the probabilities $P\left(X_{i}=1\right)=x \in[0,1]$, for $1 \leq i \leq n$. The inputs $z_{0}, \ldots, z_{n}$ are independent stochastic bit streams $Z_{0}, \ldots, Z_{n}$ representing the probabilities $P\left(Z_{i}=1\right)=b_{i} \in[0,1]$, for
$0 \leq i \leq n$, where the $b_{i}$ 's are the Bernstein coefficients. The output of the circuit is a stochastic bit stream $Y$ in which the probability of a bit being one equals the Bernstein polynomial $B(x)=\sum_{i=0}^{n} b_{i} B_{i, n}(x)$. A circuit implementation of the above example is shown in Fig. 6] [8].

The tanh and the exponentiation functions, as shown in equations (1) and (2), can also be implemented by this Bernstein polynomialbased approach. However, it requires more hardware than the FSMbased ones. To leverage the low-cost and low-power properties of the FSM-based stochastic computation, this paper demonstrates how to synthesize sophisticated functions based on a new FSM topology. Based on this technique, more sophisticated functions in ANNs, communication systems, and digital signal processing could be computed on the stochastic bit streams with lower hardware cost, lower energy consumption, and higher fault-tolerance.
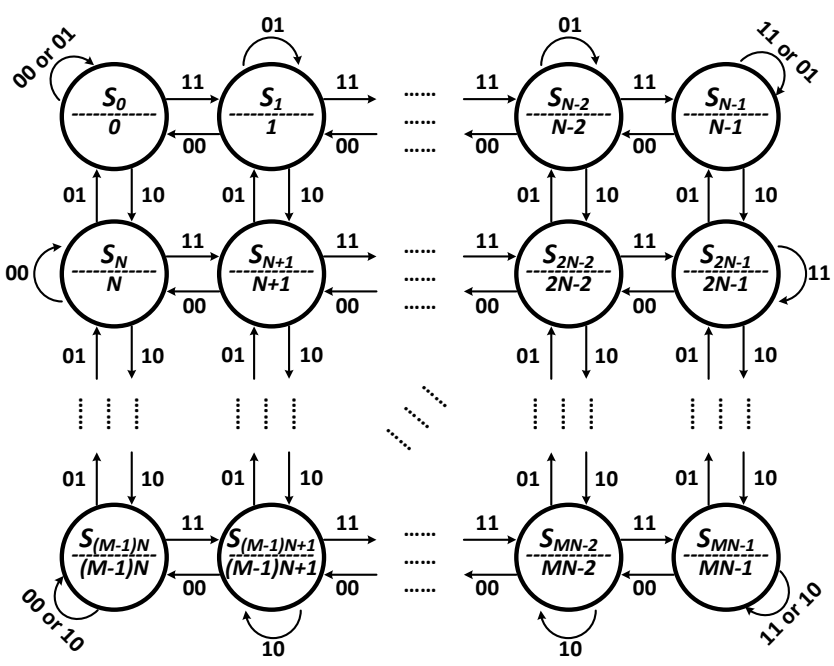

Fig. 7. The FSM has two inputs $X$ and $K$. The numbers on each arrow represent the transition condition, with the first corresponding to the input $X$ and the second corresponding to the input $K$. The FSM has $\log _{2}\lceil M N\rceil$ outputs, encoding a value in binary radix. In the figure, the number below each state $S_{t}(0 \leq t \leq M N-1)$ represents the value encoded by the outputs of the FSM when the current state is $S_{t}$. 


\section{THE NEW FSM TOPOLOGY}

The state transition diagram of the proposed FSM is shown in Fig. 7 It has two inputs (we call them $X$ and $K$ ) and in total $M \times N$ states, arranged as an $M \times N$ two-dimensional array. We normally set $M \times N=2^{R}$, where $R$ is a positive integer, because we can implement an FSM with $2^{R}$ states by $R$ D flip-flops (DFFs). In addition, we set $M=2^{\left\lfloor\frac{R}{2}\right\rfloor}$, and $N=2^{\left\lceil\frac{R}{2}\right\rceil}$. The FSM shown in Fig. 7 is a Moore-style machine, which has $\log _{2}\lceil M N\rceil$ outputs encoding an integer in the range $[0, M N-1]$ using binary radix. If the current state is $S_{t}(0 \leq t \leq M N-1)$, the value encoded by its output is just the current state number $t$. In short, we say that the output of the FSM is $t$. It can be seen that the output configuration of the FSM looks like an up/down counter. However, the state transitions of this FSM are quite different from a conventional up/down counter. For example, if we assume that the current state is $S_{N+1}$ in Fig. 7 its next state and the corresponding output will be: $S_{N+2}$ and $N+2$, if $(X, K)=(1,1) ; S_{N}$ and $N$, if $(X, K)=(0,0) ; S_{2 N+1}$ and $2 N+1$, if $(X, K)=(1,0) ; S_{2}$ and 2 , if $(X, K)=(0,1)$. An example of such an FSM with 8 states is shown in Fig. 8

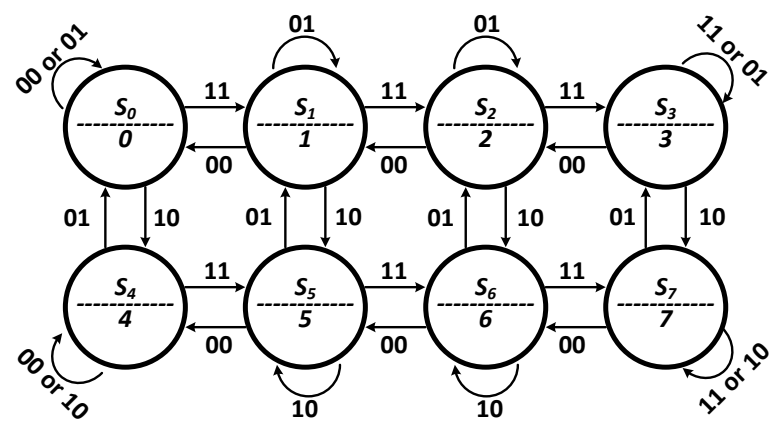

Fig. 8. An example of the state transition digram of the proposed FSM with 8 states. Here, $M=2$ and $N=4$.

The inputs $X$ and $K$ of this FSM consist of stochastic bit streams. We define the probability that each bit in the input stream $X$ is one to be $P_{X}$, and the probability that each bit in the input stream $K$ is one to be $P_{K}$. Because both inputs $X$ and $K$ are stochastic bit streams with fixed probabilities, the state transition process of the FSM can be modeled as a time-homogeneous Markov chain. It can be shown that this Markov chain is irreducible and aperiodic. Then, based on the theory of Markov chain, the FSM has an equilibrium state distribution [9]. We define the probability that the current state is $S_{t}(0 \leq t \leq M N-1)$ in the equilibrium (or the probability that the current output is $t$ ) to be $P_{t}$. Intuitively, $P_{t}$ is a function of both $P_{X}$ and $P_{K}$. In the following, we derive a closed form expression of $P_{t}$ in terms of $P_{X}$ and $P_{K}$. This expression is used to synthesize a given target function $T\left(P_{X}\right)$ on $P_{X}$. The synthesis details will be discussed in the next section.

In the following discussion, we define $i=\left\lfloor\frac{t}{N}\right\rfloor$ and $j=t$ modulo $N$, i.e., $i$ and $j$ are the quotient and the remainder of $t$ divided by $N$, respectively (or $t=i \times N+j$ ). Based on the theory of Markov chains [9], at the equilibrium stage, the probability of transitioning from the state $S_{i \times N+j}$ to its horizontal adjacent state $S_{i \times N+j-1}$, equals the probability of transitioning from the state $S_{i \times N+j-1}$ to the state $S_{i \times N+j}$. Thus, we have

$$
P_{i \times N+j} \cdot\left(1-P_{X}\right) \cdot\left(1-P_{K}\right)=P_{i \times N+j-1} \cdot P_{X} \cdot P_{K} .
$$

In addition, the probability of transitioning from the state $S_{i \times N+j}$ to its vertical adjacent state, $S_{(i-1) \times N+j}$, equals the probability of transitioning from the state $S_{(i-1) \times N+j}$ to the state $S_{i \times N+j}$ :

$$
P_{i \times N+j} \cdot\left(1-P_{X}\right) \cdot P_{K}=P_{(i-1) \times N+j} \cdot P_{X} \cdot\left(1-P_{K}\right) .
$$

Because all the individual state probabilities $P_{i \times N+j}$ (or $P_{t}$ ) must sum to unity, we have

$$
\sum_{t=0}^{M N-1} P_{t}=\sum_{i=0}^{M-1} \sum_{j=0}^{N-1} P_{i \times N+j}=1
$$

Based on equation (4), (5), and (6), we obtain

$$
P_{t}=P_{i \times N+j}=\frac{t_{x}^{i} \cdot t_{y}^{j}}{\sum_{u=0}^{M-1} \sum_{v=0}^{N-1} t_{x}^{u} \cdot t_{y}^{v}},
$$

where $t_{x}$ and $t_{y}$ are,

$$
\begin{aligned}
t_{x} & =\frac{P_{X}}{1-P_{X}} \cdot \frac{P_{K}}{1-P_{K}}, \\
t_{y} & =\frac{P_{X}}{1-P_{X}} \cdot \frac{1-P_{K}}{P_{K}} .
\end{aligned}
$$

It can be seen that equation (7) is a closed form expression of $P_{t}$ in terms of $P_{X}$ and $P_{K}$. In the next section, we discuss how $P_{t}$ is used to synthesize a given target function.

\section{THE FSM-BASED SYNTHESIS APPROACH}

In this section, we introduce how to synthesize a target function $T\left(P_{X}\right)$ based on the proposed FSM. More specifically, we use the circuit shown in Fig. 9 to synthesize $T\left(P_{X}\right)$.

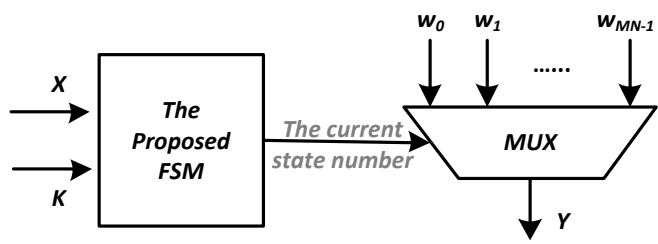

Fig. 9. The circuit for synthesizing target functions

In Fig. 9 as we introduced in the previous section, the inputs of the proposed FSM are $X$ and $K$, its output is the current state number, which is connected to the selection inputs of the multiplexer " $M U X$ ", which has $M \times N$ data inputs $\left(w_{0}, w_{1}, \cdots, w_{M N-1}\right)$. Note that if the current state of the FSM is $S_{t}(0 \leq t \leq M N-1)$, then the channel that connects $w_{t}$ to $Y$ will be selected in the "MUX."

We let $X, K$, and $w_{t}$ be stochastic bit streams, and define $P_{X}$ to be the probability of ones in $X, P_{K}$ to be the probability of ones in $K, P_{w_{t}}$ to be the probability of ones in $w_{t}$, and $P_{Y}$ to be the probability of ones in $Y$. Based on the circuit shown in Fig. 9, it can be seen that the probability that the "MUX" input $w_{t}$ is selected as its 
output is $P_{t}$, because this probability is the same as the probability that the current state of the FSM is $S_{t}$. Thus, we can obtain $P_{Y}$ as

$$
\begin{aligned}
P_{Y} & =P(Y=1) \\
& =\sum_{t=0}^{M N-1} P\left(Y=1 \mid w_{t} \text { is selected }\right) \cdot P\left(w_{t} \text { is selected }\right) \\
& =\sum_{t=0}^{M N-1} P\left(w_{t}=1\right) \cdot P\left(w_{t} \text { is selected }\right)=\sum_{t=0}^{M N-1} P_{w_{t}} \cdot P_{t} .
\end{aligned}
$$

Note that $P_{Y}$ is a function of $P_{X}, P_{K}$, and $P_{w_{t}}$, because $P_{t}$ is a function of $P_{X}$ and $P_{K}$ (refer to (7)). It can be seen that equation (8) is a closed form expression of $P_{Y}$. This expression is used to synthesize the given function $T\left(P_{X}\right)$. We define the approximation error $\epsilon$ as

$$
\epsilon=\int_{0}^{1}\left(T\left(P_{X}\right)-P_{Y}\right)^{2} \cdot d\left(P_{X}\right) .
$$

The synthesis goal is to compute $P_{w_{t}}$ and $P_{K}$ to minimize $\epsilon$. In the following, we discuss how to obtain these parameters.

A. How to Compute $P_{w_{t}}$

By expanding [9], we can rewrite $\epsilon$ as

$$
\begin{aligned}
\epsilon & =\int_{0}^{1} T\left(P_{X}\right)^{2} \cdot d\left(P_{X}\right)-2 \int_{0}^{1} T\left(P_{X}\right) \cdot P_{Y} \cdot d\left(P_{X}\right) \\
& +\int_{0}^{1} P_{Y}^{2} \cdot d\left(P_{X}\right) .
\end{aligned}
$$

The first term $\int_{0}^{1} T\left(P_{X}\right)^{2} \cdot d\left(P_{X}\right)$ is a constant because $T\left(P_{X}\right)$ is given. Thus minimizing $\epsilon$ is equivalent to minimizing the following objective function $\varphi$ :

$$
\varphi=\int_{0}^{1} P_{Y}^{2} \cdot d\left(P_{X}\right)-2 \int_{0}^{1} T\left(P_{X}\right) \cdot P_{Y} \cdot d\left(P_{X}\right) .
$$

We define a vector $\mathbf{b}$, a vector $\mathbf{c}$, and a matrix $\mathbf{H}$ as follows,

$$
\begin{gathered}
\mathbf{b}=\left[P_{w_{0}}, P_{w_{1}}, \cdots, P_{w_{M N-1}}\right]^{T}, \\
\mathbf{c}=\left[\begin{array}{c}
-\int_{0}^{1} T\left(P_{X}\right) \cdot P_{0} \cdot d\left(P_{X}\right) \\
-\int_{0}^{1} T\left(P_{X}\right) \cdot P_{1} \cdot d\left(P_{X}\right) \\
\vdots \\
-\int_{0}^{1} T\left(P_{X}\right) \cdot P_{M N-1} \cdot d\left(P_{X}\right)
\end{array}\right], \\
\mathbf{H}=\left[\mathbf{H}_{\mathbf{0}}, \mathbf{H}_{\mathbf{1}}, \cdots, \mathbf{H}_{\mathrm{MN}-\mathbf{1}}\right]^{T},
\end{gathered}
$$

where $P_{t}(0 \leq t \leq M N-1)$ in vector $\mathbf{c}$ are defined by equation $(7)$ and $H_{t}(0 \leq t \leq M N-1)$ in matrix $\mathbf{H}$ is a row vector defined as follows,

$$
\mathbf{H}_{\mathbf{t}}=\left[\begin{array}{c}
\int_{0}^{1} P_{t} \cdot P_{0} \cdot d\left(P_{X}\right) \\
\int_{0}^{1} P_{t} \cdot P_{1} \cdot d\left(P_{X}\right) \\
\vdots \\
\int_{0}^{1} P_{t} \cdot P_{M N-1} \cdot d\left(P_{X}\right)
\end{array}\right]^{T} .
$$

Note that (refer to the expression of $P_{Y}$ in (8)),

$$
\mathbf{b}^{T} \mathbf{H b}=\int_{0}^{1} P_{Y}^{2} \cdot d\left(P_{X}\right)
$$

$$
\mathbf{c}^{T} \mathbf{b}=-\int_{0}^{1} T\left(P_{X}\right) \cdot P_{Y} \cdot d\left(P_{X}\right),
$$

Thus, the objective function $\varphi$ in 10 can be rewrite as

$$
\varphi=\mathbf{b}^{T} \mathbf{H} \mathbf{b}+2 \mathbf{c}^{T} \mathbf{b} .
$$

We notice that, computing $P_{w_{t}}$ (i.e., the vector b) to minimize $\varphi$ in the form of equation (11) is a typical constrained quadratic programming problem, if $P_{K}$ is a constant. This is because $P_{t}$ is a function of both $P_{X}$ and $P_{K}$ (please refer to (7)). When we set $P_{K}$ to a constant, the integral of $P_{t}$ on $P_{X}$ is also a constant, so are the vector $\mathbf{c}$ and the matrix $\mathbf{H}$. Based on (11), the solution of $P_{w_{t}}$ (i.e., the vector b) can be obtained using standard techniques [11]. Then $P_{K}$ can be solved using a numerical approach, which will be discussed in the next section.

\section{B. How to Compute $P_{K}$}

As we introduced in the previous section, $P_{K}$ is first set to a constant. Then we compute $P_{w_{t}}$ using standard techniques [11] to minimize $\epsilon$ (or the equivalent $\varphi$ ). Note that all the values between 0 and 1 (with a step 0.001) will be used to set $P_{K}$ in the synthesis process. More specifically, we first set $P_{K}$ to 0.001 , and compute the corresponding $P_{w_{t}}$ and $\epsilon$. Next, we set $P_{K}$ to 0.002 , and compute the corresponding $P_{w_{t}}$ and $\epsilon$. So on and so forth. Finally, we set $P_{K}$ to 1 , and compute the corresponding $P_{w_{t}}$ and $\epsilon$. Among these 1000 results of $\epsilon$, we select the minimum one, and the corresponding $P_{K}$ and $P_{w_{t}}$.

\section{Summary of the Proposed Synthesis Approach}

After we get the optimal values of $P_{K}$ and $P_{w_{t}}$, the stochastic bit streams $K$ and $w_{t}$ in Fig. 9 will be generated to implement the target function $T\left(P_{X}\right)$ stochastically. Note that the synthesis approach discussed in this section also works for other FSM topologies. For example, we can use the same approach to synthesize the exponentiation and tanh functions proposed by Brown and Card [7] based on the FSM topology shown in Fig. 2[12], [13], [14]. However, that FSM topology cannot be used to synthesize more sophisticated functions, such as high order polynomials and other non-polynomials. We also tried other different FSM topologies in both single input and two inputs configurations. Only the one proposed in this paper works. Intuitively, the additional input $K$ and the balanced state transitions give the proposed FSM more degree of design freedom to synthesize more sophisticated functions.

In the next section, we use three examples to show the synthesis effect of the proposed approach. Then we compare this work to the previous ones in terms of hardware cost, energy consumption, and fault-tolerance.

\section{EXPERIMENTAL RESULTS}

In our experiments, we first present three examples to show the synthesis results of the proposed approach. Then we compare the proposed approach to the Bernstein polynomial-based approach and the binary radix representation-based approach in terms of hardware area, performance, energy consumption, and fault-tolerance. We use an 8-state FSM (i.e., the one shown in Fig. 8 ) for all the experiments.

\section{A. Synthesis Examples}

Example 1: Synthesizing Gaussian distribution function:

$$
T(x)=\frac{1}{\delta \sqrt{2 \pi}} e^{-\frac{(x-\mu)^{2}}{2 \delta^{2}}}, \quad(-1 \leq x \leq 1) .
$$




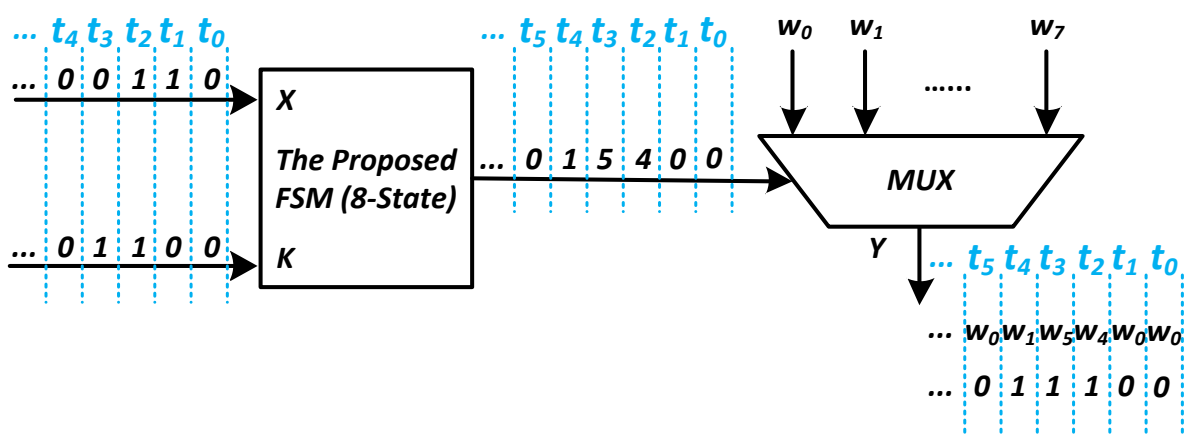

Fig. 10. An example about how the circuit shown in Fig. 9 works (the FSM is implemented with the state transition diagram shown in Fig. 8).

Because $x$ could be negative values, we need to convert $x$ to $P_{X}$ using bipolar coding (please refer to Section $\Pi$ II), i.e., we set $P_{X}=$ $0.5(x+1)$, and rewrite the target function as

$$
T\left(P_{X}\right)=\frac{1}{\delta \sqrt{2 \pi}} e^{-\frac{\left(2 P_{X}-1-\mu\right)^{2}}{2 \delta^{2}}}, \quad\left(0 \leq P_{X} \leq 1\right)
$$

Note that in $12, \delta$ could be any value as long as $\delta \geq \frac{1}{\sqrt{2 \pi}}$, because in computation on stochastic bit streams, the output is a probability value that should be greater than or equal to 0 and less than or equal to 1 and when $\delta \geq \frac{1}{\sqrt{2 \pi}}$, we guarantee that the maximal value of the function $T\left(P_{X}\right)$ is less than or equal to 1 . We normally set $\mu=0$ for simplicity, because using different value of $\mu$ will only shift the curve along on the $\mathrm{x}$-axis instead of changing the shape of the curve. If we set $\delta=2$ and $\mu=0$, for example, we compute $P_{K}$ and $P_{w_{t}}$ using the synthesis approach discussed in the previous section. The results are listed in Table I] The approximation error $\epsilon$ (defined in 9) is $7.0 \times 10^{-4}$. Fig. 11 shows the simulation result.

TABLE I

$P_{K}$ AND $P_{w_{t}}(0 \leq t \leq 7)$ FOR SYNTHESIZING THE TARGET FUNCTION IN (12) WITH $\delta=2$ AND $\mu=0$.

\begin{tabular}{l|l|l|l}
\hline \multicolumn{4}{c}{$P_{K}=0.5$} \\
\hline \hline$P_{w_{0}}=0$ & $P_{w_{1}}=1$ & $P_{w_{2}}=1$ & $P_{w_{3}}=1$ \\
$P_{w_{4}}=1$ & $P_{w_{5}}=1$ & $P_{w_{6}}=1$ & $P_{w_{7}}=0$ \\
\hline
\end{tabular}

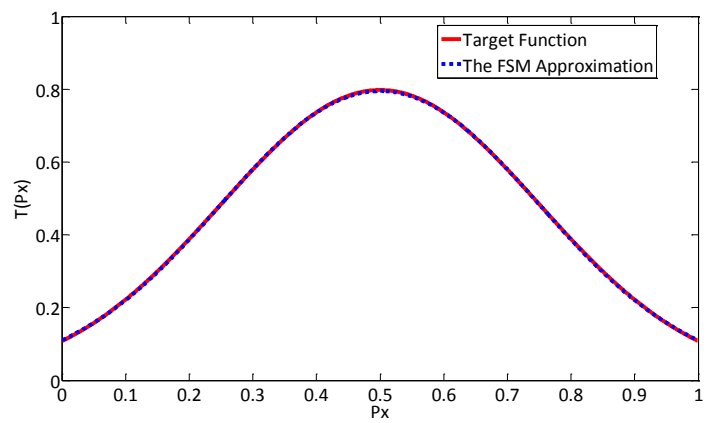

Fig. 11. Synthesis result of the target function in 12, with $\delta=2$ and $\mu=0$.
This means that, using the circuit shown in Fig. 9 if the probability of ones in the input bit stream $K$ equals 0.5 and the probabilities of ones in the input bit streams $w_{t}$ equal $P_{w_{t}}$ shown in Table I the probability of ones in the output bit stream $Y$ will be

$$
\frac{1}{2 \sqrt{2 \pi}} e^{-\frac{\left(2 P_{X}-1\right)^{2}}{8}}, \quad\left(0 \leq P_{X} \leq 1\right),
$$

where $P_{X}$ is the probability of ones in the input $X$. Note that if $P_{w_{t}}$ equal 0 or 1 , then $w_{t}$ can be set to a constant ' 0 ' or ' 1 ' correspondingly, and the hardware implementation can be further simplified.

We give an example in Fig. 10 to show how this circuit works. Assume that the circuit starts working at clock cycle $t_{0}$ and the initial state of the FSM is $S_{0}$ (please note that the initial state has no influence on the final results, it could be any one of the 8 states). The output of the FSM at $t_{0}$ is 0 (because its initial state is $S_{0}$ ) and the output of the multiplexer "MUX" at $t_{0}$ is $w_{0}$ (because its selection input equals 0 at $t_{0}$ ), and $w_{0}=0$ based on Table 1

Because at $t_{0}, X=K=0$ and the initial state is $S_{0}$, in the next clock cycle $t_{1}$, the current state of the FSM is still $S_{0}$ (and the output of the FSM is still 0) based on the state transition diagram shown in Fig. 8 The output of the "MUX" at $t_{1}$ is still $w_{0}$, which equals 0 based on Table I

In the next clock cycle $t_{2}$, based on the state transition diagram shown in Fig. 8 the current state becomes $S_{4}$ because $X=1 \mathrm{~K}=0$ at the previous clock cycle $t_{1}$, and the output of the FSM is 4 . Thus, the output of the "MUX" at $t_{2}$ becomes $w_{4}$, which equals 1 based on Table 【

So on and so forth. Assume that we use 1024 bits to represent a value stochastically. After 1024 clock cycles, if the probability of ones in $X$ equals $P_{X}$, and the probability of ones in $K$ equals 0.5 , then the probability of ones in $Y$ will be $\frac{1}{2 \sqrt{2 \pi}} e^{-\frac{\left(2 P_{X}-1\right)^{2}}{8}}$

Example 2: Synthesizing the function $\phi(x)$ used in low-density parity-check decoding [15]:

$$
\phi(x)=\log \frac{e^{x}+1}{e^{x}-1}, \quad(x>0) .
$$

For this example, we use unipolar coding because we do not deal with negative values, and set $P_{X}=x / \alpha$, where $\alpha$ is a scaling factor to map the range of $x$ to unitary. We rewrite the target function in 
terms of $P_{X}$ as

$$
T\left(P_{X}\right)=\log \frac{e^{\alpha P_{X}}+1}{e^{\alpha P_{X}}-1}, \quad\left(0<P_{X} \leq 1\right) .
$$

If we set $\alpha=20$, for example, we compute $P_{K}$ and $P_{w_{t}}$ using the proposed synthesis approach and show the results in Table II] The approximation error $\epsilon$ (defined in (9) is $2.0 \times 10^{-4}$. Fig. 12 shows the simulation result.

TABLE II

$P_{K}$ AND $P_{w_{t}}$ FOR SYNTHESIZING THE TARGET FUNCTION IN 13 with $\alpha=20$.

\begin{tabular}{c|c|c|c}
\hline \multicolumn{4}{c}{$P_{K}=0.9375$} \\
\hline \hline$P_{w_{0}}=1$ & $P_{w_{1}}=0$ & $P_{w_{2}}=0$ & $P_{w_{3}}=0$ \\
$P_{w_{4}}=1$ & $P_{w_{5}}=0$ & $P_{w_{6}}=0$ & $P_{w_{7}}=0$ \\
\hline
\end{tabular}

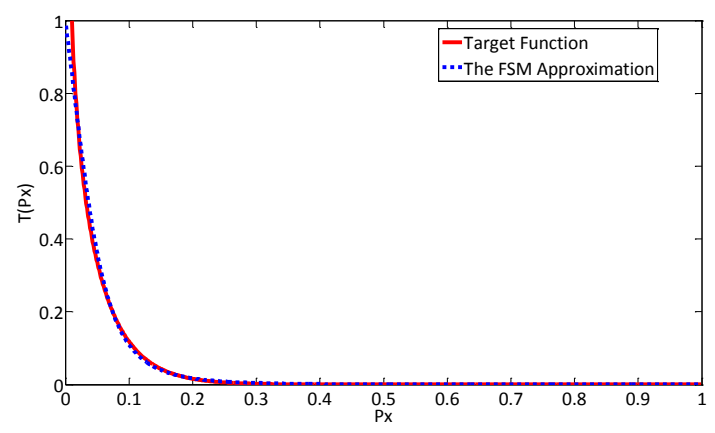

Fig. 12. Synthesis result of the target function in 13 with $\alpha=20$.

Example 3: Synthesizing the following high order polynomial $\lambda(x)$ used in low-density parity-check coding [16]:

$$
\begin{aligned}
\lambda(x) & =0.1575 x+0.3429 x^{2}+0.0363 x^{5}+0.059 x^{6} \\
& +0.279 x^{8}+0.1253 x^{9}, \quad(0 \leq x \leq 1) .
\end{aligned}
$$

For this example, we use unipolar coding because we do not deal with negative values and $x$ is in the unitary range. We rewrite the target function in terms of $P_{X}\left(P_{X}=x\right)$ as

$$
\begin{aligned}
T\left(P_{X}\right) & =0.1575 P_{X}+0.3429 P_{X}^{2}+0.0363 P_{X}^{5}+0.059 P_{X}^{6} \\
& +0.279 P_{X}^{8}+0.1253 P_{X}^{9}, \quad\left(0 \leq P_{X} \leq 1\right) .
\end{aligned}
$$

We compute $P_{K}$ and $P_{w_{t}}$ using the proposed synthesis approach and show the results in Table III The approximation error $\epsilon$ (defined in (9)) is $4.0 \times 10^{-6}$. Fig. 13 shows the simulation result. Note that the bit streams $w_{1}$ and $w_{5}$ can be generated with extremely low cost using the technique proposed by Qian et al. [17.

TABLE III

$P_{K}$ AND $P_{w_{t}}$ FOR SYNTHESIZING THE TARget FUnCTION IN 14.

\begin{tabular}{l|l|l|l}
\hline \multicolumn{4}{c}{$P_{K}=0.1875$} \\
\hline \hline$P_{w_{0}}=0$ & $P_{w_{1}}=0.86$ & $P_{w_{2}}=0$ & $P_{w_{3}}=0$ \\
$P_{w_{4}}=0$ & $P_{w_{5}}=0.89$ & $P_{w_{6}}=0$ & $P_{w_{7}}=1$ \\
\hline
\end{tabular}

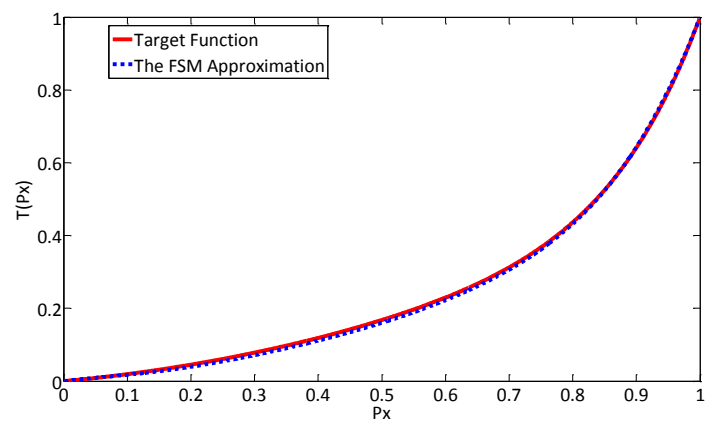

Fig. 13. Synthesis result of the target function in 14.

\section{B. Comparison with the Bernstein Polynomial-Based Approach}

As we introduced in Section III the Bernstein polynomial-based approach uses combinational logic (an adder and a multiplexer, as shown in Fig. 6 to perform computation on stochastic bit streams. Hardware area required by this approach depends on the degree of polynomial. Table IV lists its area in terms of the number of fan-in two logic gates $[8]$.

TABLE IV

THE NUMBER OF THE FAN-IN TWO LOGIC GATES FOR COMPUTING BERNSTEIN POLYNOMIALS OF DEGREE 3, 4, 5, AND 6 [8].

\begin{tabular}{c||c|c|c|c}
\hline Degree $n$ & 3 & 4 & 5 & 6 \\
\hline Number of Gates & 22 & 40 & 49 & 58 \\
\hline
\end{tabular}

Note that by using the 8-state FSM shown in Fig. 8, we can synthesize a polynomial of degree up to 9 (refer to Example 3). In addition, for those non-polynomials, such as the target functions introduced in Example 1 and Example 2, the Bernstein polynomialbased approach normally takes at least degree 6 to obtain the same level of approximation error. The proposed FSM with 8 states can be implemented using 3 D-flip-flops (DFFs) as follows,

$$
\begin{aligned}
& D_{2}=X K Q_{0}+X Q_{1}+K Q_{1}+Q_{1} Q_{0}, \\
& D_{1}=X \bar{K}+X Q_{2}+\bar{K} Q_{2}, \\
& D_{0}=\bar{X} \bar{K} Q_{1} \bar{Q}_{0}+\bar{X} K Q_{0}+X K Q_{1}+X \bar{K} Q_{0}+X K Q_{0},
\end{aligned}
$$

where $D_{0}, D_{1}$, and $D_{2}$ are the inputs of the three DFFs, and $Q_{0}, Q_{1}$, and $Q_{2}$ are the corresponding outputs. Because it is a Moore FSM, we assign $Q_{2} Q_{1} Q_{0}=000$ for state $S_{0}, Q_{2} Q_{1} Q_{0}=001$ for state $S_{1}, \cdots$, and $Q_{2} Q_{1} Q_{0}=111$ for state $S_{7}$. Based on the report of the logic synthesis tool Synonsys Design Compiler, the entire circuit (including the multiplexer in Fig. 9) can be implemented using 45 fan-in two logic gates. Please note that the evaluation is based on a generalized version of the circuit shown in Fig. 9 if $w_{t}$ is set to a constant ' 0 ' or ' 1 ', the circuit can be further simplified and the number of logic gates can be further reduced. It can be seen that, to synthesize non-polynomials and polynomials of degree greater than 4, the proposed FSM takes less hardware.

In terms of performance, because both techniques compute on stochastic bit streams, they have equivalent processing time. In terms of energy consumption, we assume that given a CMOS technology, a digital circuit consumes a constant power dissipation per unit area. We use the product of area and processing time as a metric of the energy consumption [7]. Because these two techniques have 
equivalent processing time, the FSM-based approach consumes less energy when computing non-polynomials and high order polynomials with degree larger than 4 .

In terms of fault-tolerance, we compare the two techniques when the input data is corrupted with noise. We evaluate the fault-tolerant performance on circuits implementing the target functions introduced in the last section and other functions such as trigonometric functions $(\sin (x), \cos (x)$, and $\tan (x))$ and logarithmic functions $\left(y=\log _{2}(x), y=\log _{10}(x)\right.$, and $\left.y=\ln (x)\right)$. The length of the stochastic bit streams which are used to represent a value is set to 1024. We define the error ratio $\gamma$ as the percentage of random bit flips that occur in the computation. We choose the error ratio $\gamma$ to be $0 \%, 0.5 \%, 1 \%, 5 \%$, and $10 \%$. For example, under $10 \%$ error ratio, 102 of 1024 bits will be flipped in the computation. To measure the impact of the noise, we evaluated each target function at 13 distinct input data points: $0.2,0.25,0.3, \cdots, 0.8$. For each error ratio $\gamma$, each target function, and each evaluation point, we simulated both the FSM-based implementation and the Bernstein polynomial-based implementation 1000 times. We averaged the relative errors over all simulations. Finally, for each error ratio $\gamma$, we averaged the relative errors over all target functions and all evaluation points. Table $\mathrm{V}$ shows the average relative error of the two different implementations versus different $\gamma$ values. It can be seen that these two techniques has almost equivalent fault-tolernace (the difference is less than $0.5 \%$ ), because both techniques perform computation on stochastic bit streams.

TABLE V

RELATIVE ERROR FOR THE FSM-BASED IMPLEMENTATION AND THE BERNSTEIN POLYNOMIAL-BASED IMPLEMENTATION OF TARGET FUNCTION COMPUTATION VERSUS THE ERROR RATIO $\gamma$ IN THE INPUT DATA.

\begin{tabular}{c||c|c|c|c|c}
\hline Error Ratio $\gamma(\%)$ & 0 & 0.5 & 1 & 5 & 10 \\
\hline \hline Relative Error of the FSM (\%) & 2.26 & 2.78 & 3.16 & 6.75 & 11.2 \\
\hline Relative Error of Bernstein (\%) & 2.21 & 2.72 & 3.36 & 6.25 & 11.7 \\
\hline
\end{tabular}

\section{Comparison with the Binary Radix-Based Approach}

Assume that $M$ is the number of bits used to represent a numerical value in binary radix. In order to get the same resolution for computation on stochastic bit streams, we need a $2^{M}$-bit stream to represent the same value. Both Qian et al. [8] and Brown et al. [7] showed that, when $M \leq 10$, computation on stochastic bit streams has better performance than the ones based on binary radix using adders and multipliers in terms of hardware area and energy consumption. In fact, in most applications of the stochastic computation, $M$ is between 8 to 10 [7], [8]. As we discussed in the Section $\mathrm{V}-\mathrm{B}$, the proposed approach using FSM has better performance than the one proposed by Qian et al. [8] for computing non-polynomials and high order polynomials. Thus, when $M \leq 10$, the proposed approach also has better performance than the ones using binary radix for those functions. In addition, computing on stochastic bit streams offers tunable precision: as the length of the stochastic bit stream increases, the precision of the value represented by it also increases. Thus, without hardware redesign, we have the flexibility to trade-off precision and computation time. The main issue of this computing technique is the long latency. However, it can be solved by using a faster clock frequency, because its logic is simple and has shorter critical path. Parallel computing can also be used to solve this issue. For example, in digital image processing applications, we can process multiple pixels in an image in parallel. In ANN applications, we can process the computation on multiple neurons in parallel.

\section{CONCLUSION}

This paper proposed a new FSM topology to synthesize computation on stochastic bit streams for complex and useful functions. Compared to other implementations, the resulting circuits are less costly in terms of hardware area and energy consumption. In future work, we will study synthesis techniques for general purpose computation using these techniques. Our eventual goal is a fully stochastic design of a microprocessor.

\section{ACKNOWLEDGMENT}

This work is supported in part by National Science Foundation grant NO. CCF-1241987. Any opinions, findings and conclusions or recommendations expressed in this material are those of the authors and do not necessarily reflect the views of the NSF. This work is also supported in part by the Minnesota Supercomputing Institute and a donation from NVIDIA. The authors would like to thank the reviewers for their helpful feedback.

\section{REFERENCES}

[1] N. Iqbal, M. Siddique, and J. Henkel, "Seal: soft error aware low power scheduling by monte carlo state space under the influence of stochastic spatial and temporal dependencies," in Design Automation Conference (DAC), 2011 48th ACM/EDAC/IEEE, pp. 134-139, IEEE, 2011.

[2] X. Shih, H. Lee, K. Ho, and Y. Chang, "High variation-tolerant obstacleavoiding clock mesh synthesis with symmetrical driving trees," in Proceedings of the International Conference on Computer-Aided Design, pp. 452-457, IEEE Press, 2010.

[3] S. Rehman, M. Shafique, F. Kriebel, and J. Henkel, "Reliable software for unreliable hardware: embedded code generation aiming at reliability," in Proceedings of the seventh IEEE/ACM/IFIP international conference on Hardware/software codesign and system synthesis, pp. 237-246, ACM, 2011.

[4] P. Li and D. J. Lilja, "A low power fault-tolerance architecture for the kernel density estimation based image segmentation algorithm," in IEEE International Conference on Application - specific Systems, Architectures and Processors, ASAP'11, 2011.

[5] P. Li and D. J. Lilja, "Using stochastic computing to implement digital image processing algorithms," in IEEE International Conference on Computer Design, ICCD'11, 2011.

[6] B. Gaines, "Stochastic computing systems," Advances in Information Systems Science, vol. 2, no. 2, pp. 37-172, 1969.

[7] B. D. Brown and H. C. Card, "Stochastic neural computation I: Computational elements," IEEE Transactions on Computers, vol. 50, pp. 891905, September 2001.

[8] W. Qian and M. D. Riedel, "The synthesis of robust polynomial arithmetic with stochastic logic," in 45th ACM/IEEE Design Automation Conference, DAC'08, pp. 648-653, 2008.

[9] A. A. Markov, "Extension of the limit theorems of probability theory to a sum of variables connected in a chain," reprinted in Appendix B of: R. Howard. Dynamic Probabilistic Systems, volume 1: Markov Chains. John Wiley and Sons, 1971.

[10] G. Lorentz, Bernstein Polynomials. University of Toronto Press, 1953.

[11] G. Golub and C. Van Loan, Matrix computations, vol. 3. Johns Hopkins Univ Pr, 1996.

[12] P. Li, W. Qian, M. Riedel, K. Bazargan, and D. Lilja, "The synthesis of linear finite state machine-based stochastic computational elements," in Design Automation Conference (ASP-DAC), 2012 17th Asia and South Pacific, pp. 757-762, IEEE, 2012.

[13] P. Li, D. J. Lilja, W. Qian, and K. Bazargan, "Using a two-dimensional finite-state machine for stochastic computation," in International Workshop on Logic and Synthesis, IWLS'12, 2012.

[14] P. Li, W. Qian, and D. J. Lilja, "A stochastic reconfigurable architecture for fault-tolerant computation with sequential logic," in IEEE International Conference on Computer Design, ICCD'12, 2012.

[15] W. Ryan, "An introduction to ldpc codess," 2003.

[16] T. Richardson, M. Shokrollahi, and R. Urbanke, "Design of capacityapproaching irregular low-density parity-check codes," Information Theory, IEEE Transactions on, vol. 47, no. 2, pp. 619-637, 2001.

[17] W. Qian, M. Riedel, K. Bazargan, and D. Lilja, "The synthesis of combinational logic to generate probabilities," in Proceedings of the 2009 International Conference on Computer-Aided Design, pp. 367 374, ACM, 2009. 